\title{
Primer registro de la comestibilidad de Phillipsia domingensis Berk. (Pezizales: Ascomycota): aspectos nutricionales y actividad biológica
}

\section{First record of the edibility of Phillipsia domingensis Berk. (Pezizales:} Ascomycota): nutritional aspects and biological activity

\author{
Jesús Jiménez-Zárate', Roberto Garibay-Orijel², Elhadi M. Yahia ${ }^{3}$, Edgardo Ulises Esquivel-Naranjo ${ }^{4}$, Fausto \\ Arellano-Carbajal ${ }^{5}$, Fidel Landeros ${ }^{1}$ \\ ${ }^{1}$ Laboratorio de Micología, ${ }^{3}$ Laboratorio de Fitoquímicos y Nutrición Humana, ${ }^{4}$ Laboratorio de Microbiología Molecular, \\ ${ }^{5}$ Laboratorio de Genética Molecular, Facultad de Ciencias Naturales, Universidad Autónoma de Querétaro. Carretera a \\ Chichimequillas s/n, Ejido Bolaños, C.P. 76140, Santiago de Querétaro, Querétaro, México. \\ ${ }^{2}$ Instituto de Biología, Universidad Nacional Autónoma de México. Tercer Circuito s/n, Ciudad Universitaria, C.P. 04510, México, \\ D.F., México.
}

\section{RESUMEN}

Antecedentes: Los hongos silvestres comestibles son un alimento de alto valor nutricional y se les considera una fuente potencial de compuestos bioactivos. Actualmente, no existen reportes sobre la comestibilidad de Phillipsia domingensis.

Objetivo: Registrar por primera vez el uso de esta especie en la cultura náhuatl de la Huasteca Potosina, así como algunos de sus aspectos nutricionales y actividad biológica.

Métodos: El estudio etnográfico se realizó en San Luis Potosí, México. Los componentes nutricionales se determinaron por análisis químico proximal, la capacidad antioxidante mediante los métodos DPPH y FRAP, la actividad antifúngica por inhibición radial del micelio de tres hongos patógenos y la actividad antihelmíntica por la parálisis de Caenorhabditis elegans.

Resultados y conclusiones: Se registra la comestibilidad, comercio, uso medicinal y nombres tradicionales de $P$. domingensis. Los parámetros nutricionales están en el promedio reportado para otros hongos. La actividad biológica se consideró con alta capacidad antioxidante, baja actividad antifúngica y prometedora antihelmíntica. Este hongo tiene potencial como alimento funcional, convirtiéndolo en un recurso alimentario con cualidades para ser aprovechado en otras regiones donde se distribuye.

Palabras clave: náhuatl, etnomicología, aporte nutricional, capacidad antioxidante, actividad antifúngica, actividad antihelmíntica

\section{ABSTRACT}

Background: Wild edible mushrooms are high nutritional value foods and are considered potential sources of bioactive compounds. Currently, there are no reports on the edibility of Philipsia domingensis.

Objective: To present the first record of this fungus in the nahua culture of the Huasteca Potosina, as well as some of its nutritional and biological activity properties.

Methods: The ethnographic study was carried out in San Luis Potosí, México. Nutritional components were determined by proximal chemical analysis, the antioxidant capacity by DPPH and FRAP methods, the antifungal activity by radial inhibition of the mycelium of three pathogenic fungi, and the anthelmintic activity by the paralysis of Caenorhabditis elegans.

Results and conclusions: Edibility, trade, medicinal use and traditional names of $P$. domingensis were recorded. Nutritional parameters are within the average values reported for other fungi. Its biological activity was considered with high in antioxidant capacity, low for antifungal activity and promising for anthelmintic one. We state that this species has potential as a functional food, making it a food resource with qualities that can be used in other regions where it is distributed. Keywords: nahuatl, ethnomycology, nutritional contribution, antioxidant capacity, antifungal activity, anthelmintic activity

\section{ARTICLE HISTORY}

Received 02 July 2019 / Accepted 18 March 2020

Published on line: 30/05/2020

\section{CORRESPONDING AUTHOR}

\4. Fidel Landeros, landeros@uaq.mx

ORCID: 0000-0002-9861-2042 


\section{INTRODUCCIÓN}

Los hongos silvestres comestibles proporcionan un beneficio económico a las personas o familias que los colectan (Boa, 2005) y son un alimento de alto valor nutricional con sabores únicos y ricos en proteínas, carbohidratos y ácidos grasos insaturados (Colak et al., 2009). Asimismo, son considerados como alimentos funcionales por sus actividades benéficas al organismo, debido a moléculas como $\beta$-glucanos, ergosterol y antioxidantes. De estos últimos, se estima que los macromicetos son ricos en actividad antioxidante (Boda et al., 2012), la cual está relacionada principalmente a su contenido de fenoles totales, que a su vez podría depender de su localización geográfica y eventual condición de estrés, entre otros factores (Puttaraju et al., 2006). Se sabe también que varias especies de hongos silvestres comestibles tienen propiedades terapéuticas, muchas derivadas de componentes celulares y metabolitos secundarios (Boa, 2005). Entre los compuestos que se pueden obtener de los hongos son de gran interés los fungicidas y fungistáticos (Wen-Hsiung et al., 2010), así como los que tienen la capacidad de inmovilizar y/o matar nematodos (Degenkolb y Vilcinskas, 2016). Diversos trabajos reportan la actividad antifúngica (Radhajeyalakshmi et al., 2012; Al-Fatimi et al., 2013) y antihelmíntica en macromicetos (Degenkolb y Vilcinskas, 2016), por lo que se consideran una fuente potencial de estos compuestos.

A nivel mundial, la mayoría de los hongos consumidos pertenecen a los basidiomicetos (Boa, 2005) y de Ascomicetos, Pezizales, resaltan diversas especies de Morchella (Liu et al., 2018) y las trufas (Patel, 2012), las cuales son apreciadas y consumidas por varias culturas alrededor del mundo, por lo que tienen una gran importancia económica. Dentro de Sarcoscyphaceae (Pezizales) se han registrado especies de relevancia etnomicológica como Cookeina (García et al., 2014) y Sarcoscypha (Garibay-Orijel et al., 2010), utilizadas como alimento en algunas zonas tropicales del mundo. Sin embargo, del género Phillipsia no se tiene ningún reporte de comestibilidad en México (Garibay-Orijel y Ruan-Soto, 2014), Latinoamérica (Garibay-Orijel et al., 2010) y el mundo (Boa, 2005); de acuerdo con Guzmán (2003) estos hongos no tienen propiedades culinarias. La mayoría de los trabajos sobre el género Phillipsia se refieren a su filogenia y taxonomía, donde se han descrito alrededor de 28 especies a nivel mundial (De- nison, 1969; Hansen et al., 1999; Calonge et al., 2006). Phillipsia domingensis (Berk.) Berk. ex Denison, es la especie con mayor número de registros en el mundo con amplia distribución en los trópicos e incluso representada en la filatelia de Islas de Navidad, Australia y Fiji en 1984. Por otra parte, la notable variabilidad de colores en el himenio y análisis filogenéticos han demostrado que podría tratarse de un complejo de especies (Hansen et al., 1999).

En los últimos años en México, se ha registrado el consumo de hongos silvestres previamente desconocido (Robles-García et al., 2016; Rodríguez-Hernández, 2018), utilizados principalmente por pueblos originarios. La curva de acumulación de especies comestibles no ha llegado a su asíntota y por ende, se esperan nuevos registros (Garibay-Orijel y Ruan-Soto, 2014).

El objetivo del presente estudio es registrar por primera vez el consumo tradicional de Phillipsia domingensis en la cultura náhuatl de San Luis Potosí, así como analizar su valor nutricional, capacidad antioxidante, actividad antifúngica y antihelmíntica.

\section{MATERIALES Y MÉTODOS}

\section{Área de estudio}

El estudio se llevó a cabo en el sureste de San Luis Potosí, México, específicamente en los municipios de Matlapa y Axtla de Terrazas (Figura 1) con vegetación de bosque tropical perennifolio, bosque tropical subdeciduo y pastizal inducido (Rzedowski, 1961). El $79 \%$ de la población es de ascendencia indígena, náhuatl en su mayoría (CDI-INEGI, 2010) y de acuerdo con SEDESOL (2014), en Axtla de Terrazas el $25 \%$ o más de la población se encuentra en pobreza extrema y Matlapa con alto grado de marginación.

\section{Estudio etnográfico}

Se visitaron 20 localidades de Axtla de Terrazas y 20 de Matlapa, donde por medio del uso de ejemplares y fotografías se les preguntó a las personas sobre $P$. domingensis. Se compraron ascomas en el mercado municipal de Matlapa y otros colectados en campo en los alrededores de las comunidades de San José Barrio Arriba, Tamala y Tlaxco, acompañados de las personas del lugar. La determinación taxonómica se hizo con base en las estructuras macro- y microscópicas siguiendo a Calonge et al. (2006) y Hansen et al. (1999). 


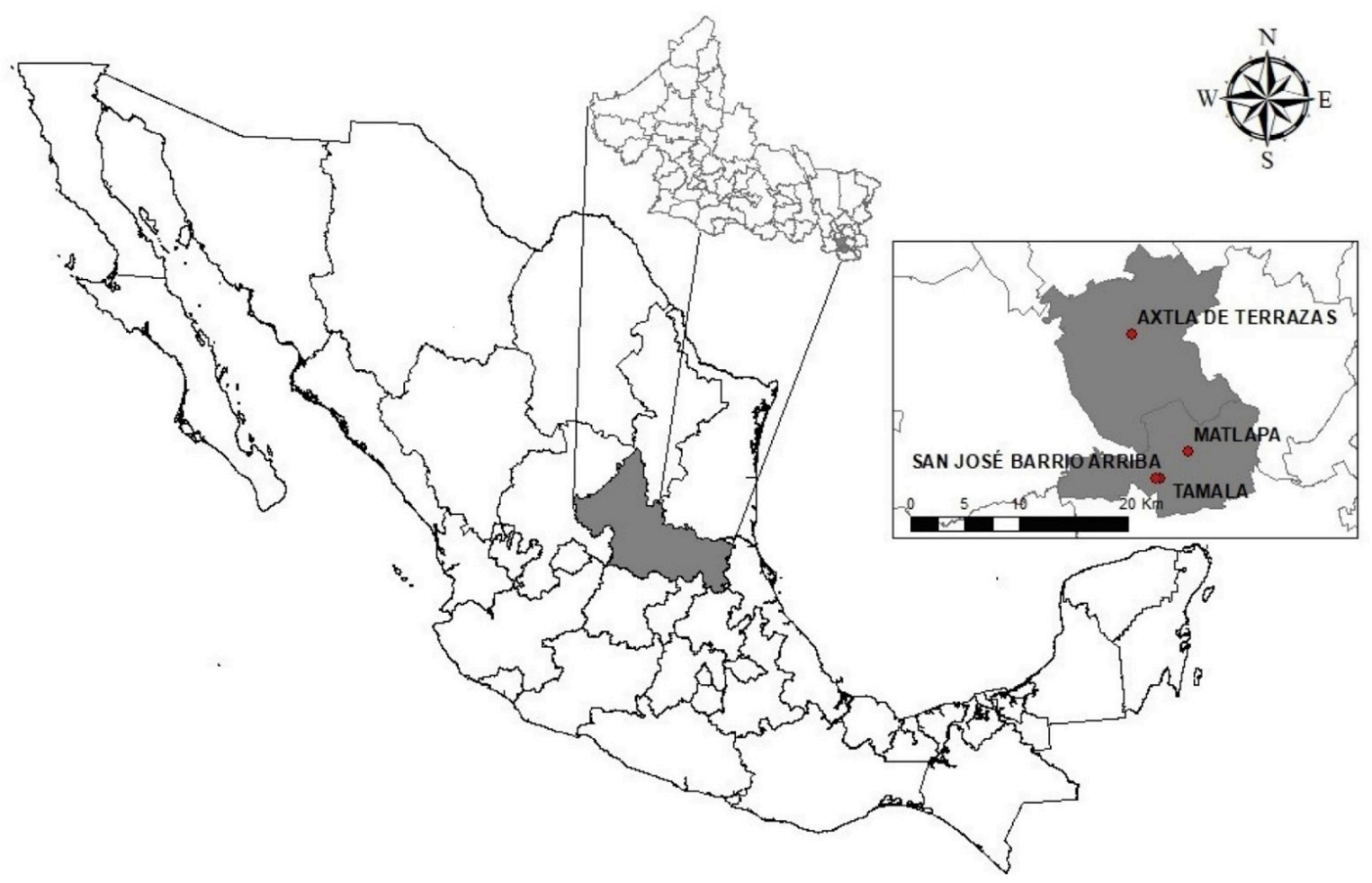

Figura 1. Área de estudio. Cabeceras municipales Axtla de Terrazas y Matlapa, y localidades San José Barrio Arriba y Tamala, Matlapa, San Luis Potosí.

\section{Procesado de muestras}

Para los análisis en laboratorio se obtuvieron aproximadamente $1.2 \mathrm{~kg}$ de ascomas de $P$. domingensis, los cuales se envolvieron en hojas de papatla (Heliconia schiedeana), colocados en una hielera con bloques congelados y transportados a la Universidad Autónoma de Querétaro (UAQ). Los hongos se congelaron en nitrógeno líquido, se liofilizaron por $72 \mathrm{~h}$ y pulverizaron con un molino. La harina obtenida se almacenó en frascos ámbar rotulados y sellados con una película plástica hasta su uso.

\section{Análisis químicos proximales}

Los componentes nutricionales se evaluaron de acuerdo con los métodos oficiales de AOAC (1990). Se calcularon en porcentajes los siguientes parámetros: humedad, materia seca, cenizas, proteína cruda, fibra cruda, extracto etéreo y extracto libre de nitrógeno en base seca (bs) y húmeda (bh). Cada prueba se hizo por triplicado y se realizó un análisis de medias de Tukey-Kramer con un nivel de significancia de 0.05.

\section{Capacidad antioxidante}

A partir del material liofilizado se prepararon extractos lipofílicos donde se detectaron compuestos como ca- rotenoides y en los hidrofílicos principalmente fenoles de acuerdo con el método de Wu et al. (2004) modificado por Corral-Aguayo et al. (2008). Para la curva de calibración se preparó una solución stock $2 \mathrm{mM}$ de Trolox, con la cual se hizo una dilución seriada con un total de 10 concentraciones. La capacidad antioxidante se midió por la prueba de inhibición del radical DPPH y el poder antioxidante de reducción férrica, FRAP. Para DPPH se siguió el método de Kim et al. (2002), modificado por Corral-Aguayo et al. (2008), el cual consistió en preparar una solución DPPH/metanol a una concentración de $100 \mu \mathrm{M}$. Para FRAP se aplicó el método de Benzie y Strain (1996), modificado por Szöllösi y Szöllösi-Varga (2002), el cual consistió en preparar una solución FRAP (búfer de acetato de sodio/TPTZ/FeCl3 [10:1:1 v/v/v]).

Para ambas pruebas se utilizaron microplacas de 96 pozos (Becton Microtest Tissue Culture). En ambos ensayos se colocó primero $300 \mu \mathrm{L}$ del blanco, posteriormente se llenaron todos los pozos requeridos con $280 \mu \mathrm{L}$ de solución DPPH y FRAP respectivamente; se adicionaron $20 \mu \mathrm{L}$ de cada concentración de trolox y del extracto, todo por triplicado y se dejó reposar 30 min en la oscuridad. La lectura se hizo a 492 y 630 $\mathrm{nm}$ para DPPH y FRAP respectivamente, en un espec- 
trofotómetro (SpectraMAX 250 microplate spectrophotometer) con el programa SPF. Los resultados se expresaron en " $\mu \mathrm{mol}$ equivalente de Trolox / $100 \mathrm{~g}$ de hongo" y en "mg equivalente de Trolox / $100 \mathrm{~g}$ de hongo" en base seca (bs) y húmeda (bh) para ambos métodos. Con los resultados obtenidos se hizo un análisis de medias de Tukey-Kramer con un nivel de significancia de 0.05 .

\section{Actividad antifúngica}

Se determinó con un ensayo de inhibición del crecimiento radial del micelio, en el que se confrontó el extracto de $P$. domingensis y el antifúngico benomilo (ANTRAK 50), contra dos hongos fitopatógenos: Fusarium oxysporum y Rhizoctonia solani y un microparásito Trichoderma atroviride. Estas cepas se encuentran almacenadas en el Laboratorio de Biología Molecular de Microorganismos de la Facultad de Ciencias Naturales, Universidad Autónoma de Querétaro.

Para el bioensayo se preparó un extracto metanólico de $P$. domingensis, porque se considera uno de los mejores solventes para extraer sustancias antifúngicas (Radhajeyalakshmi et al., 2012) y se elaboró de acuerdo al método de Ko et al. (2010) con una concentración final del extracto de $200 \mathrm{mg} / \mathrm{mL}$. Se agregaron $200 \mu \mathrm{L}$ del extracto en cajas Petri de $90 \times 15 \mathrm{~mm}$ con medio agar papa dextrosa (PDA), con cultivos de 2 a 5 d de los hongos que se confrontaron; se generaron discos de $5 \mathrm{~mm}$ de diámetro y se colocaron en el centro de la caja Petri previamente inoculada, mientras que para el testigo una caja sin inocular. La inoculación del extracto antifúngico y sembrado se hizo por triplicado por hongo confrontado. Se incubaron a $28{ }^{\circ} \mathrm{C}$ y se dejaron crecer hasta que el micelio del testigo estuvo ca. a $3 \mathrm{~mm}$ del margen de la caja. Los cultivos se fotografiaron y con el programa ImageJ se midió el crecimiento micelial en los tratamientos vs. testigo (100\%), todo por triplicado. Se promediaron los porcentajes de inhibición del extracto y el antifúngico para cada hongo evaluado y se hizo un análisis de medias de Tukey-Kramer con un nivel de significancia de 0.05 .

\section{Actividad antihelmíntica}

Se evaluó acorde con el procedimiento de Piña-Vázquez et al. (2017), en el que se confrontó el extracto metanólico de $P$. domingensis contra el nematodo Caenorhabditis elegans. Se utilizó una placa de 24 po- zos donde a cada pozo a utilizar se le agregaron 250 $\mu \mathrm{L}$ de medio $\mathrm{M9}$, luego se agregaron 10 nematodos por pozo y $250 \mu \mathrm{L}$ de extracto metanólico de $P$. domingensis por triplicado. Como control negativo se utilizó medio M9 y como positivo Levamisol, antihelmíntico comercial, ambos controles también se realizaron por triplicado. La revisión de los pozos se hizo de manera aleatoria a las 1, 2, 4, 6, 8, 24 y 48 h donde se contó el número de nematodos paralizados bajo un microscopio estereoscópico. Cada nematodo representó el 10 \% en cada pozo evaluado; así, la actividad antihelmíntica se midió en porcentaje representada por los nematodos paralizados en su totalidad cuyos resultados se promediaron.

\section{RESULTADOS Y DISCUSIÓN}

\section{Etnográficos}

En el mercado municipal de Matlapa se encontraron comerciantes de hongos silvestres comestibles de los alrededores entre los cuales se identificó $P$. domingensis, mezclado con Cookeina sulcipes, C. tricholoma y Auricularia delicata, cuyo precio venta de este grupo fue de $\$ 10.00 \mathrm{mn}$ (Figura 2). Estas personas (hongueros) y los hongos comercializados provenían de San José Barrio Arriba y Tamala (Figura 1). El número de vendedores observados en las diferentes visitas fue de dos a cuatro solo en los días de tianguis. Comentaron que difícilmente colectaban cantidades considerables de $P$. domingensis debido a su baja abundancia, por lo que generalmente se ofrecía combinado con otros hongos. En Axtla de Terrazas y Matlapa, P. domingensis es usado como comestible, difiriendo con lo reportado por otros estudios en los que se citó como tóxica (Jiménez-González, 2008), no comestible (Garza et al., 2009) y medicinal o para teñir (Ruan-Soto, 2014), siendo el primer reporte sobre la comestibilidad de la especie.

En la mayoría de las comunidades de Axtla de Terrazas, los informantes refirieron que tienen varios años sin consumirlo porque ya no crece en los alrededores. Esta escasez o ausencia lo atribuyen principalmente a la deforestación, ya que esta especie crece en selvas poco perturbadas, lo que resalta su importancia ecológica como indicador biológico (Guzmán, 1994). En Matlapa donde hay zonas mejor conservadas, los pobladores aún lo consumen, aunque poco frecuentemente debido a su escasez y algunas personas pre- 


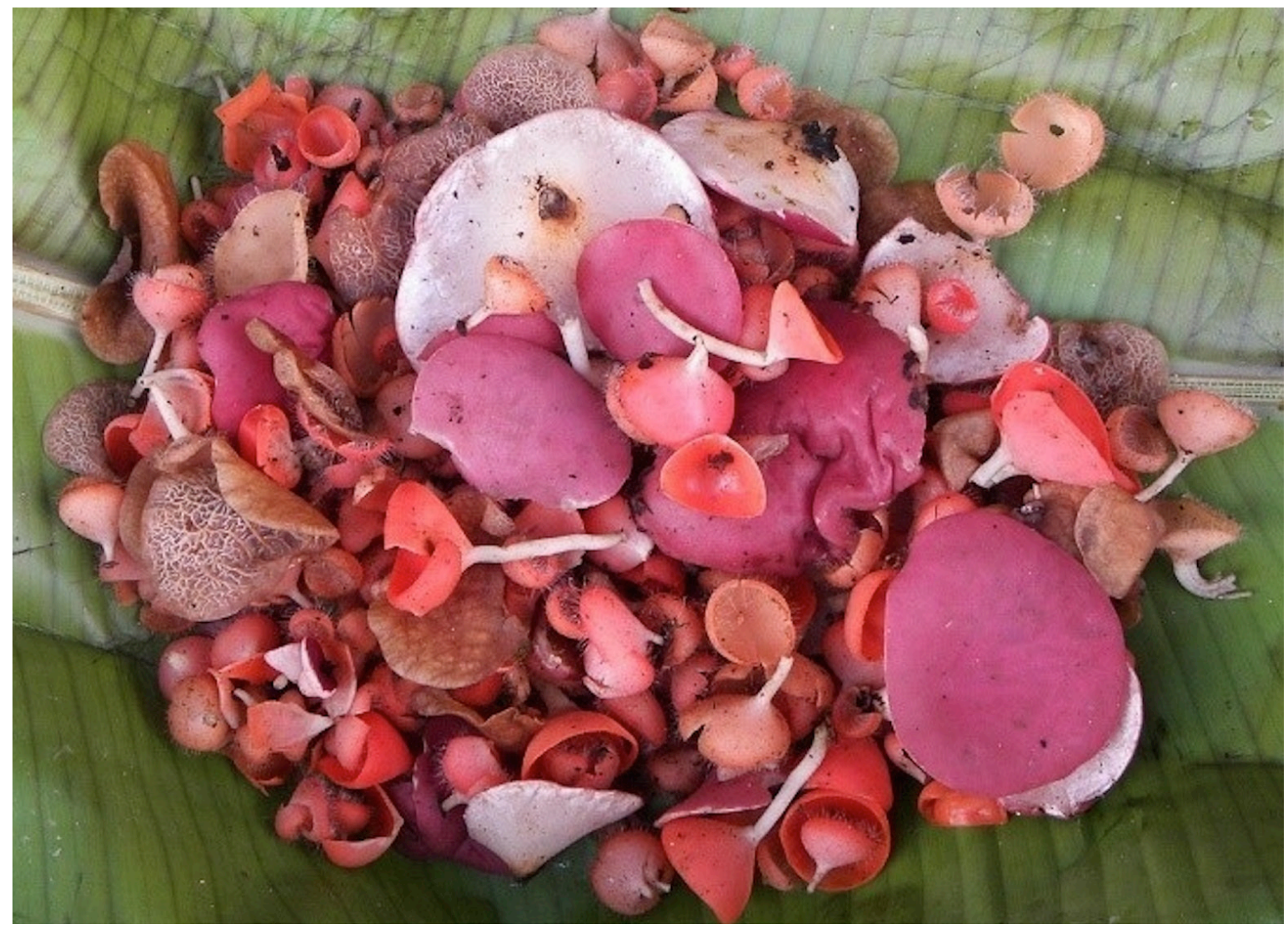

FIGURA 2. Unidad de hongos a la venta en el mercado municipal de Matlapa. Phillipsia domingensis mezclada con Cookeina sulcipes, C. tricoloma y Auricularia delicata.

fieren dejárselos a las ardillas. Aunque se encuentra gran parte del año, es más común en octubre sobre palos podridos asociado con $C$. sulcipes y $C$. tricholoma.

Phillipsia domingensis no tiene alguna manera especial de prepararse porque generalmente está mezclado con otros hongos. La mayoría de los informantes enfatizó que "no le entra la sal", lo cual no minimizó su apreciación culinaria ya que mencionaron que tiene buen sabor. Respecto a sus cualidades medicinales, un informante expresó que sirve para la urticaria y/o angioedema (ronchas). Para ello, se debe moler el hongo y agregar sal antes de untarlo. Un médico tradicional de Axtla de Terrazas lo utiliza para tratar el asma. Para tal fin, mencionó que muele los hongos, los coloca en miel como conservador y daba de tomar la esencia a los pacientes. La información recabada del uso medicinal de esta especie fue escasa e inconsistente, considerándose un conocimiento idiosincrático como en otras regiones (Johns et al.,1990).

En esta región, $P$. domingensis es conocido principalmente como chichileltlapachtli o chikinte morado. Las nominaciones tanto en náhuatl como en español son: chichileltlapachtli (hígado de perro), pesoeltlapachtli (hígado de tejón), eltlapachtli (hígado), piyoeltlapachtli (hígado de pollo), tojtoeltlapachtli (hígado de guajolote), pitsoeltlapachtli (hígado de puerco), hígado de animal, hígado de mapache, chikinte eltlapachtli (hongo de hígado), chikinte (hongo), chikinte morado (hongo morado), chikintechichiltik (hongo rojo), itemetltotolin o tojtoitemetl (molleja de guajolote), piyoitemetl (molleja de pollo), wewejitemetl o abuelojitemetl (molleja de anciano), chilnejnepilli (lengua de perro), asadura de perro, asadura de tlacuache y orejuelas. Eltlapachtli es la palabra náhuatl para referirse al hígado, mientras que itemetl para molleja. Es importante resaltar que el náhuatl de esta región es una variante de la lengua influenciada por el tének, lo cual tiene relevancia debido a que pertenecen a familias lingüísticas diferentes. Por un lado, el náhuatl se agrupa dentro de las lenguas yuto-nahua mientras que el tének a las lenguas mayenses. Asimismo, algunos nombres difieren de la gramática correcta del náhuatl y otros corresponden a mezclas de lenguas. 
En otras regiones de México también se han registrado otros nombres asignados a P. domingensis como leok, lak, chak xikin (oreja roja) y kuxum che' (hongo de árbol) en Maya Lacandón (Ruan-Soto et al., 2007, 2014). Leok y lak son nombres dados por lacandones a $P$. domingensis sin traducción al español, mientras kuxum che' es una categoría residual que incluye a todos los hongos lignícolas (Ruan-Soto com. pers.). Al comparar los nombres asignados a $P$. domingensis por los nahuas de la huasteca y los mayas lacandones, se observó que los primeros utilizan un mayor número de nombres más específicos que los mayas. La cantidad y especificidad de nombres asignados a una especie de acuerdo con Turner (1973), se correlaciona positivamente con la importancia cultural para el grupo étnico. Esta diferencia en la importancia puede derivar principalmente por la utilización de $P$. domingensis como alimento de los nahuas.

Descripción de la especie

Phillipsia domingensis (Berk.) Berk. ex Denison, Mycologia 61(2): 293 (1969)

Figura 3

Ascoma en forma de disco subcóncavo de 0.5 a $8.5 \mathrm{~cm}$ de diámetro, himenio liso de color violáceo, parte estéril lisa a ligeramente rugosa de color blanco, sésil o con un pequeño pseudoestípite, contexto de consistencia sólida de color blanco (Figura 3D). Ascas de 335-410 $\times$ 12.5-17.5 $\mu \mathrm{m}$, cilíndricas, octosporadas. Paráfisis de $2.5 \mu \mathrm{m}$ de grosor en la parte media y 2.5-3.75 $\mu \mathrm{m}$ en el ápice, hialinas y septadas (Figura 3A-C). Ascosporas de 20.8-27.2 × 12.8-15.4 $\mu \mathrm{m}$, elipsoides, hialinas, gutuladas y ornamentadas con crestas longitudinales.

Hongos lignícolas que crecen solitarios o en pequeños grupos sobre madera en descomposición y generalmente en zonas conservadas.

Material estudiado. México, San Luis Potosí, municipio de Matlapa, mercado de Matlapa, Jiménez-Zárate s/n (QMEX), octubre 3, 2014; Jiménez-Zárate 43 (QMEX), septiembre 27, 2015; Torres-Anaya 5 (QMEX), octubre 7, 2016. Tlaxco, Jiménez-Zárate 40 (QMEX), septiembre 24, 2015.

\section{Aporte nutricional}

El porcentaje de humedad de P. domingensis de 92 $\%$ es similar al reportado para otras especies de hon-
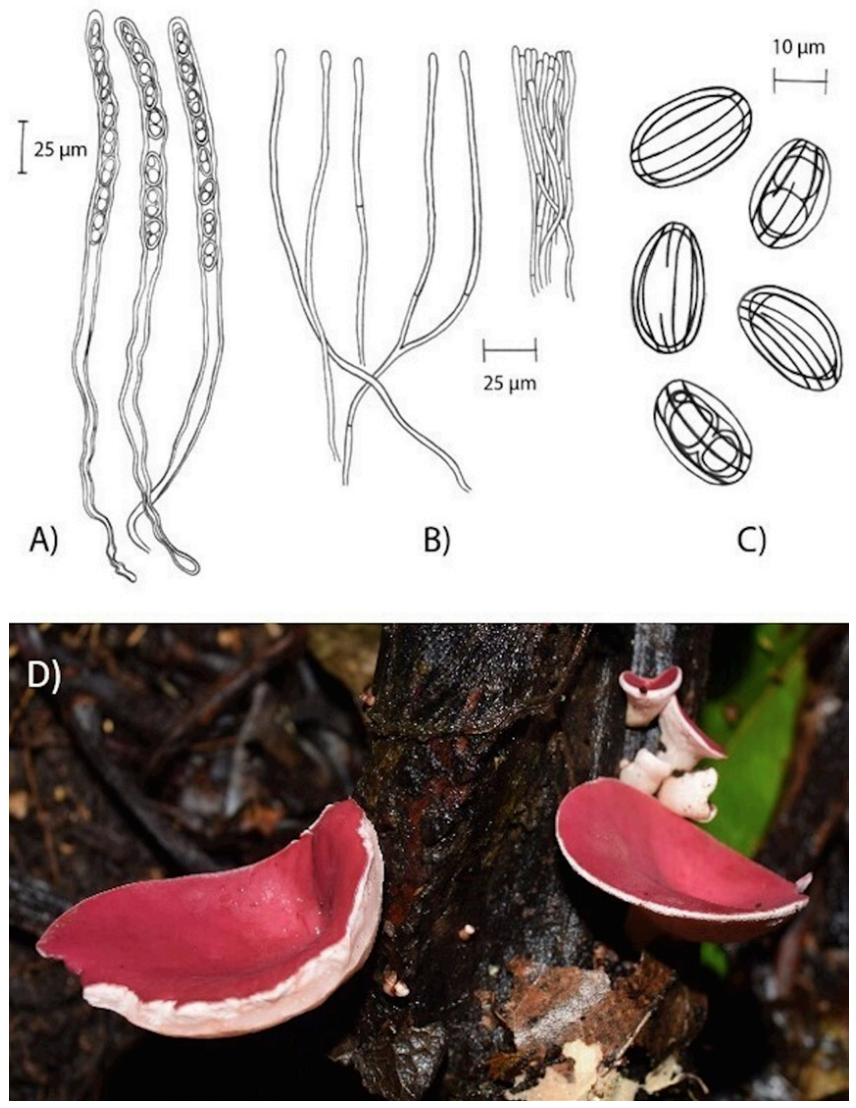

Figura 3. Phillipsia domingensis Jiménez-Zárate 43 (OMEX). A: Ascas. B: Paráfisis. C: Ascosporas. D: Ascoma.

gos (Tabla 1). El extracto libre de nitrógeno con 55 $\%$ es el mayor componente de la materia seca de $P$. domingensis y muestra el contenido de materia soluble en agua como carbohidratos, pigmentos y vitaminas. Este porcentaje es mayor al determinado para Fistulinella wolfeana (Robles-García et al., 2016), similar a Amanita caesarea (Ouzouni et al., 2009) y menor a Ramaria flava (Colak et al., 2009). En proteína y fibra cruda los valores de $P$. domingensis son menores comparados con hongos como Calvatia gigantea (Agrahar-Murugkar y Subbulakshmi, 2005) y F. wolfeana (Robles-García et al., 2016) y similares a Cantharellus cibarius (Agrahar-Murugkar y Subbulakshmi, 2005). En cuanto al contenido de extracto etéreo $P$. domingensis presentó un valor menor que $A$. caesarea, Volvariella volvacea, R. flava, C. gigantea y C. cibarius, hongos consumidos en varias partes del mundo. En general, P. domingensis es un hongo rico en carbohidratos, proteína y fibra, además bajo en grasa, cuyos valores promedio se encuentran dentro de los rangos reportados para otros hongos silvestres comestibles. 
TABLA 1. Parámetros nutricionales de Phillipsia domingensis expresados en porcentajes comparados con otras especies fúngicas

\begin{tabular}{|c|c|c|c|c|c|c|c|c|c|}
\hline $\begin{array}{l}\text { ESPECIE O } \\
\text { ALIMENTO }\end{array}$ & $\% \mathrm{H}$ & $\% \mathrm{MS}$ & & $\mathrm{C}$ & $\mathrm{EE}$ & $\mathrm{FC}$ & PC & ELN & REFERENCIA \\
\hline \multirow[t]{2}{*}{ Phillipsia domingensis } & 92 & 7.0 & bs & 9.9 & 1.2 & 12.6 & 20.8 & 55.6 & Este estudio \\
\hline & & & bh & 0.7 & 0.1 & 0.9 & 1.5 & 3.9 & \\
\hline \multirow[t]{2}{*}{ Fistulinella wolfeana } & 73 & 26.3 & bs & 4.5 & 0.7 & 23 & 29.8 & 30.2 & Robles-García et al. (2016) \\
\hline & & & bh & 1.5 & 0.5 & 2.9 & 3.4 & 18.0 & \\
\hline \multirow[t]{2}{*}{ Amanita caesarea } & 90 & 9.4 & bs & 6.1 & 3.5 & -- & 34.8 & 55.6 & Ouzouni et al. (2009) \\
\hline & & & bh & -- & -- & -- & -- & -- & \\
\hline \multirow[t]{2}{*}{ Ramaria flava } & 93 & 6.1 & bs & 3.1 & 5.2 & -- & 35.6 & 65 & Colak et al. (2009) \\
\hline & & & bh & -- & -- & -- & -- & -- & \\
\hline \multirow[t]{2}{*}{ Volvariella volvaceae } & 91 & 9 & bs & 10 & 3.3 & 9.8 & 28 & 6.5 & Mshandete y Cuff (2007) \\
\hline & & & bh & -- & -- & -- & -- & -- & \\
\hline \multirow[t]{2}{*}{ Calvatia gigantea } & -- & 4.4 & bs & 6.3 & 1.0 & 22.0 & 23.7 & & Agrahar-Murugkar y Sub- \\
\hline & & & bh & -- & -- & -- & -- & -- & bulakshmi (2005) \\
\hline \multirow[t]{2}{*}{ Cantharellus cibarius } & -- & 15.9 & bs & 13.3 & 1.6 & 12.8 & 21.1 & & \\
\hline & & & $\mathrm{bh}$ & -- & -- & -- & -- & -- & \\
\hline
\end{tabular}

\% H: porcentaje de humedad. \% MS: porcentaje de materia seca. bs: base seca. bh: base húmeda. C: cenizas. EE: extracto etéreo. FC: fibra cruda. PC: proteína cruda. ELN: extracto libre de nitrógeno.

\section{Capacidad antioxidante}

En DPPH y FRAP, la fracción hidrofílica de P. domingensis aportó 98.3 y $93.6 \%$ vs. el extracto lipofílico 1.7 y $6.3 \%$ respectivamente de la actividad antioxidante total (Tabla 2). La actividad antioxidante de los hongos está ligada principalmente a su contenido de fenoles (Puttaraju et al., 2006) en la fracción hidrofílica. La actividad antioxidante total en $\mu$ mol equivalente de Trolox/100g de hongo determinada por DPPH fue de $65400 \pm 2247$ bs y $4604 \pm 158$ bh, mientras que en el método FRAP $40401 \pm 2281$ bs y $2844 \pm 160.62$ bh. En comparación con algunos frutos, los cuales son considerados en general como alimentos ricos en antioxi- dantes, en ambos métodos $P$. domingensis con 4604 y $2844 \mu \mathrm{mol} / 100 \mathrm{~g}$ bh en DPPH y FRAP respectivamente, estuvo por debajo de la guayaba (Psidium guajava) con 6230 y $7500 \mu \mathrm{mol} / 100 \mathrm{~g}$ bh en DPPH y FRAP respectivamente, lo cual era esperado. Aún así, P. domingensis tiene buenos valores superiores a la fresa (Fragaria vesca) con 1209 y $2300 \mu \mathrm{mol} / 100 \mathrm{~g}$ bh en DPPH y FRAP, el mango (Mangifera indica) con 1328 y $1500 \mu \mathrm{mol} / 100 \mathrm{~g}$ bh DPPH y FRAP, y la papaya (Carica papaya) con 421 y $1000 \mu \mathrm{mol} / 100 \mathrm{~g}$ bh en DPPH y FRAP respectivamente (Corral-Aguayo et al., 2008). Al igual que otros hongos (Boda et al. 2012), P. domingensis podría usarse como un alimento funcional con beneficios a la salud.

TABLA 2. Actividad antioxidante de fracción lipofílica e hidrofílica de Phillipsia domingensis expresados en $\mu \mathrm{mol} / 100 \mathrm{~g}$ y $\mathrm{mg} / 100 \mathrm{~g}$ equivalentes de trolox

\begin{tabular}{|c|c|c|c|c|c|}
\hline \multirow{2}{*}{\multicolumn{2}{|c|}{$\frac{\text { P. DOMINGENSIS }}{\text { MÉTODO }}$}} & \multicolumn{2}{|c|}{ EXTRACTO LIPOFÍLICO } & \multicolumn{2}{|c|}{ EXTRACTO HIDROFÍLICO } \\
\hline & & MOL/100G & $\mathrm{MG} / 100 \mathrm{G}$ & - MOL/100G & $\mathrm{MG} / 100 \mathrm{G}$ \\
\hline \multirow[t]{2}{*}{ Bs } & DPPH & $1114.3 \pm 334.3$ & $278.9 \pm 83.7$ & $64286.5 \pm 2394.32$ & $16090.3 \pm 599.3$ \\
\hline & FRAP & $2556.0 \pm 175.5$ & $639.7 \pm 43.9$ & $37845.9 \pm 2446.70$ & $9472.5 \pm 612.4$ \\
\hline \multirow[t]{2}{*}{$\mathrm{Bh}$} & DPPH & $78.4 \pm 23.5$ & $19.6 \pm 5.9$ & $4525.8 \pm 168.6$ & $1132.8 \pm 42.2$ \\
\hline & FRAP & $179.9 \pm 12.4$ & $45.0 \pm 3.1$ & $2664.4 \pm 172.3$ & $666.9 \pm 43.11$ \\
\hline
\end{tabular}

bs: base seca. bh: base húmeda. 


\section{Actividad antifúngica}

La inhibición del crecimiento de hongos patógenos por $P$. domingensis fue menor del $30 \%$, siendo 27,17 y $16 \%$ para F. oxysporum, R. solani y $T$. atroviride respectivamente (Figura 4). Nikolovska-Nedelkoska et al. (2013) encontraron también una nula o escasa actividad antifúngica en macromicetos. Contrariamente, $R$. solani fue inhibido $83 \%$ con el antifúngico Antrak 50 al $2 \%$ y en varios hongos se ha reportado actividad antifúngica (Al-Fatimi et al., 2013).

\section{Actividad antihelmíntica}

La actividad antihelmíntica se observó hasta las 24 h, paralizando un $63 \%$ de nematodos y $100 \%$ a las $36 \mathrm{~h}$. A las $48 \mathrm{~h}$ los controles cambiaron, M9 pasó de 0 a 3 \% y Levamisol de 100 a 86 \%; así, los resultados no se consideraron confiables (Figura 5). Aunque un efecto retardado no es lo ideal, el alto porcentaje de inhibición de $P$. domingensis resultó promisorio.

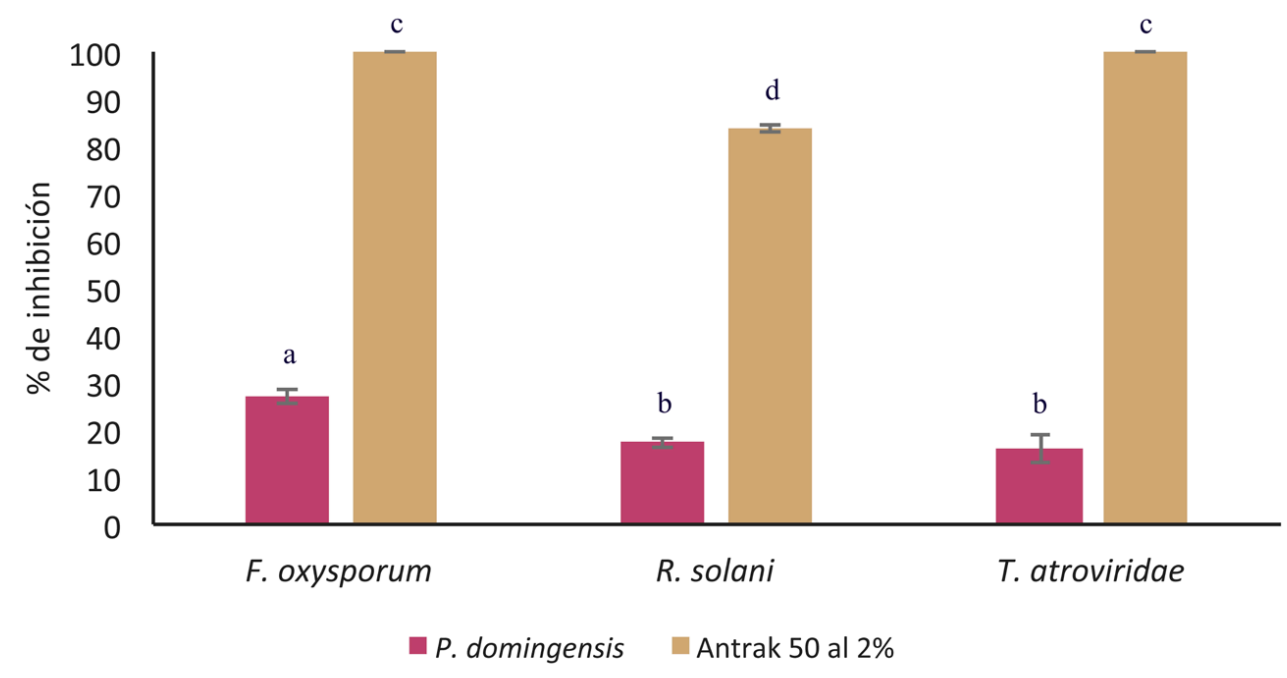

FIGURA 4. Actividad antifúngica de Phillipsia domingensis y el antifúngico benomilo (Antrak 50) contra tres hongos patógenos. Desviación estándar de medias por triplicado. Letras sobre columnas indican diferencias estadísticas de una misma categoría $(P<0.05)$.

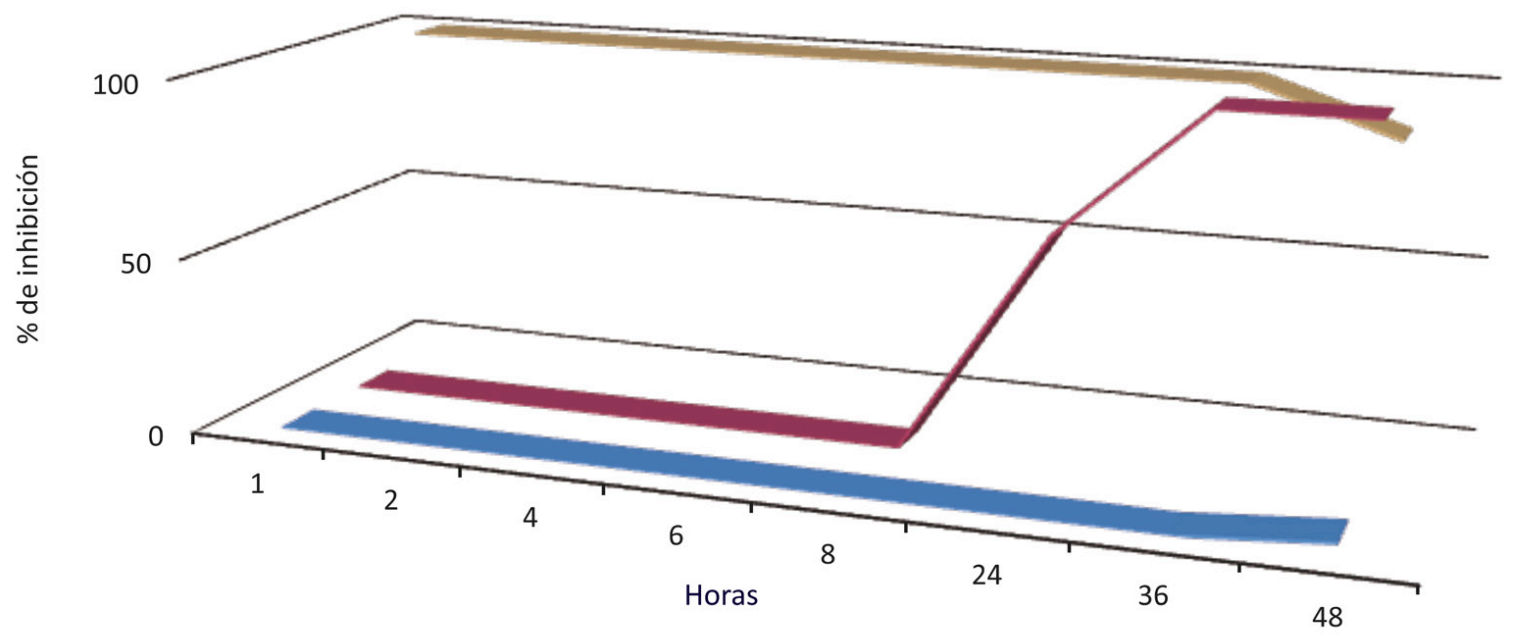

M9 $\square$. domingensis $\square$ Levamisol

Figura 5. Actividad antihelmíntica de Phillipsia domingensis contra Caenorhabditis elegans. Control negativo "medio M9", control positivo "Levamisol". Promedio en porcentaje de inhibición por triplicado. 


\section{CONCLUSIONES}

Aunque Phillipsia domingensis está ampliamente distribuida a nivel mundial es la primera vez que se reporta su comestibilidad, comercialización, medicina tradicional y nombres en lengua náhuatl, español y mixtos. Su aporte nutricional fue rico en extractos libres de nitrógeno, fibra y proteína cruda, y bajo en extracto etéreo. Su actividad antioxidante fue más alta que en algunos frutos de consumo frecuente. Aunque la actividad biológica mostró una respuesta diferenciada en la capacidad antifúngica y la actividad antihelmíntica con altos porcentajes de inhibición, se sugieren estudios a mayor profundidad para determinar su verdadero potencial y ser aprovechado en otras regiones del mundo donde se distribuye.

\section{AGRADECIMIENTOS}

AI MGIC Josué Pedro Fernando Baltazar Rivera por la elaboración del mapa. A la Universidad Autónoma de Querétaro por el proyecto FNB201820. A las personas de las comunidades que amablemente nos brindaron la información que aquí se está publicando.

\section{LITERATURA CITADA}

Agrahar-Murugkar, D., G. Subbulakshmi, 2005. Nutritional value of edible wild mushrooms collected from the Khasi hills of Meghalaya. Food Chemistry 89: 599-603. Doi: 10.1016/j.foodchem.2004.03.042

Al-Fatimi, M., G. Schröder, H. Kreisel, U. Lindequist, 2013. Biological activities of selected basidiomycetes from Yemen. Pharmazie 68: 221-226. Doi: 10.1691/ph.2013.2729

AOAC (Association of Official Analytical Chemists), 1990. Official methods of analysis. 15th ed. Association of Official Analytical Chemists, Washington.

Benzie, F.F., J.J. Strain, 1996. The ferric reducing ability of plasma (FRAP) as a measure of "antioxidant power": the FRAP assay. Analytical Biochemistry 239: 70-76. Doi: 10.1006/abio.1996.0292

Boa, E., 2005. Los hongos silvestres comestibles. Perspectiva global de su uso e importancia para la población. FAO, Roma.

Boda, R.H., A.H. Wani, M.A. Zargar, B.A. Ganie, B.A. Wani, S.A. Ganie, 2012. Nutritional values and antioxidant potential of some edible mushrooms of Kashmir valley. Pakistan Journal of Pharmaceutical Sciences 25: 623-627.

Calonge, F.D., M. Mata, L. Umaña, 2006. El género Phillipsia (Ascomycota) en Costa Rica, con una clave para identificar las especies. Boletín de la Sociedad Micológica de Madrid 30: 35-42.

CDI (Comisión Nacional para el Desarrollo de los Pueblos Indígenas), INEGI (Instituto Nacional de Estadística Geografía e Informática), 2010. Catálogo de localidades indígenas 2010. Disponible en: www.cdi.gob.mx/index.php?option=com_content\&view=article\&id=2578 [consultado 28/10/2018].
Colak, A., Ö. Faiz, E. Sesli, 2009. Nutritional composition of some wild edible mushrooms. Turkish Journal of Biochemistry 34 : 25-31.

Corral-Aguayo, R.D., E.M. Yahia, A. Carillo-Lopez, G. González-Aguilar, 2008. Correlation between some nutritional components and the total antioxidant capacity measured with six different assays in eight horticultural crops. Journal of Agricultural and Food Chemistry 56: 10498-10504. Doi: 10.1021/jf801983r

Degenkolb, T., A. Vilcinskas, 2016. Metabolites from nematophagous fungi and nematicidal natural products from fungi as alternatives for biological control, Part II: metabolites from nematophagous basidiomycetes and non-nematophagous fungi. Applied Microbiology and Biotechnology 100: 3813-3824. Doi: 10.1007/s00253-015-7234-5

Denison, W.C., 1969. Central American Pezizales. III. The genus Phillipsia. Mycologia 61: 289-304. Doi: 10.2307/3757123

García, M., A. Notario, J. Quaedvlieg, M. Cardozo, A. Cárdenas, A. Portal, 2014. Evaluación preliminar de macrohongos en seis áreas con diferente grado de perturbación en Madre de Dios. Biodiversidad Amazónica 4: 58-73.

Garibay-Orijel, R., F. Ruan-Soto, E. Estrada-Martínez, 2010. El conocimiento micológico tradicional, motor para el desarrollo del aprovechamiento de los hongos comestibles y medicinales. In: Martínez-Carrera D., N. Curvetto, M. Sobal, P. Morales, V.M. Mora (eds.), Hacia un desarrollo sostenible del sistema de producción-consumo de los hongos comestibles y medicinales en Latinoamérica: avances y perspectivas en el siglo XXI. COLPOSUNS-CONACYT-AMC-UAEM-UPAEP-IMINAP, Puebla. Pp. 243270.

Garibay-Orijel, R., F. Ruan-Soto, 2014. Listado de los hongos silvestres consumidos como alimento tradicional en México. In: Moreno-Fuentes, A., R. Garibay-Orijel (eds.), La etnomicología en México. Estado del arte. Red de Etnoecología y Patrimonio Biocultural (CONACYT)-Universidad Autónoma del Estado de Hidalgo-Instituto de Biología UNAM-Sociedad Mexicana de Micología-Asociación Etnobiológica Mexicana A.C.-Grupo Interdisciplinario para el Desarrollo de la Etnomicología en México-Sociedad Latinoamericana de Etnobiología, México. Pp. 91-112.

Garza, F., M. Quiñonez, V. Chacón, L. Garza, A. Carrillo, E. Cázares, 2009. Sustainable management of edible forest mushrooms in Chihuahua, Mexico. Actes du Colloque International sur les Champignons Forestiers Comestibles à Potentiel Commercial. Univesrité Labal, Québec. Pp. 105-118.

Guzmán, G., 1994. Algunos aspectos importantes en la ecología de los hongos (en especial de los macromicetos). Ecológica 3(2): 1-9.

Guzmán, G., 2003. Los Hongos de El Edén Quintana Roo. Introducción a la micobiota tropical de México. INECOL-CONABIO, Xalapa. Doi: 10.1590/S0036-46652004000500017

Hansen, K., D.H. Pfister, D.S. Hibbett, 1999. Phylogenetic relationships among species of Phillipsia inferred from molecular and morphological data. Mycologia 91: 299-314. Doi: $10.2307 / 3761375$ 
Jiménez-González, M., 2008. Uso de los macromicetes de Molango de Escamilla, Hidalgo, México. Tesis de Licenciatura, Universidad Autónoma del Estado de Hidalgo, Pachuca de Soto, Hidalgo.

Johns, T., J.O. Kokwaro, E.K. Kimanani, 1990. Herbal remedies of the Luo of Siaya district, Kenya: Establishing quantitative criteria for consensus. Economic Botany 44: 369-381. Doi: 10.1007/ BF03183922

Kim D.O., K.W. Lee, H.J. Lee, C.Y. Lee, 2002. Vitamin C equivalent antioxidant capacity (VCEAC) of phenolic phytochemicals. Journal of Agricultural and Food Chemistry 50: 3713-3717. Doi: 10.1021/jf020071c

Ko W.-H., Y.-J. Tsou, M.-J. Lin, L.-L. Chern, 2010. Activity and characterization of secondary metabolites produced by a new microorganism for control of plant diseases. New Biotechnology 27: 397-402. Doi: 10.1016/j.nbt.2010.05.014

Liu, Q., H. Ma, Y. Zhang, C. Dong, 2018. Artificial cultivation of true morels: current state, issues and perspectives. Critical Reviews in Biotechnology 38: 259-271. Doi: 10.1080/07388551.2017.1333082

Mshandete, A.M., J. Cuff, 2007. Proximate and nutrient composition of three types of indigenous edible wild mushrooms grown in Tanzania and their utilization prospects. African Journal of Food Agriculture Nutrition and Development 7(6): S/N Doi: 10.18697/ ajfand.17.2615

Nikolovska-Nedelkoska, D., N. Atanasova-Pančevska, H. Amedi, D. Veleska, E. Ivanova, M. Karadelev, D. Kungulovski, 2013. Screening of antibacterial and antifungal activities of selected Macedonian wild mushrooms. Matica Srpska Journal for Natural Sciences 124: 333-340. Doi: 10.2298/ZMSPN1324333N

Ouzouni, P.K., D. Petridis, W.-D. Koller, K.A. Riganakos, 2009. Nutritional value and metal content of wild edible mushrooms collected from west Macedonia and Epirus, Greece. Food Chemistry 115: 1575-1580. Doi: 10.1016/j.foodchem.2009.02.014

Patel, S., 2012. Food, health and agricultural importance of truffles: A review of current scientific literature. Current Trends in Biotechnology and Pharmacy 6: 15-27.

Piña-Vázquez, D.M., Z. Mayoral-Peña, M. Gómez-Sánchez, L.A. Salazar-Olivo, F. Arellano-Carbajal, 2017. Anthelmintic effect of Psidium guajava and Tagetes erecta on wild-type and Levamisole-resitant Caenorhabditis elegans strains. Journal of Ethnopharmacology 202: 92-96. Doi: 10.1016/j.jep.2017.03.004

Puttaraju, N.G., S.U. Venkateshaiah, S.M. Dharmesh, S.M.N. Urs, R. Somasundaram, 2006. Antioxidant activity of indigenous edible mushrooms. Journal of Agricultural and Food Chemistry 54: 9764-9772. Doi: 10.1021/jf0615707

Radhajeyalakshmi, R., R. Valazhahan, V. Prakasam, 2012. In vitro evaluation of solvent extracted compounds from edi- ble macromycetes against phytopathogenic fungi. Archives of Phytopathology and Plant Protection 45: 293-300. Doi: 10.1080/03235408.2011.559048

Robles-García, D., E.M. Yahia, J. García-Jiménez, E.U. Esquivel-Naranjo, F. Landeros, 2016. First ethnomycological record of Fistulinella wolfeana as an edible species and some of its nutritional values. Revista Mexicana de Micología 44: 31-39.

Rodríguez-Hernández, A., 2018. Aspectos bioculturales de Saproamanita thiersii (Fungi: Agaricales) en el municipio de Apaxco de Ocampo, Estado de México. Tesis de Licenciatura, Universidad Autónoma del Estado de Hidalgo, Pachuca de Soto, Hidalgo.

Ruan-Soto, F., R. Mariaca-Méndez, J. Cifuentes, F. Limón-Aguirre, L. Pérez-Ramírez, S. Sierra-Galván, 2007. Nomenclatura, clasificación y percepciones locales acerca de los hongos en dos comunidades de la Selva Lacandona, Chiapas, México. Etnobiología 5: 1-20.

Ruan-Soto, F., 2014. Micofília y micofobia: estudio comparativo de la importancia cultural de los hongos comestibles entre grupos mayas de tierras altas y de tierras bajas de Chiapas, México. Tesis de Doctorado, Universidad Nacional Autónoma de México, México, D.F.

Rzedowski, J., 1961. Vegetación del estado de San Luis Potosí. Tesis de Doctorado. Universidad Nacional Autónoma de México. México, D.F.

SEDESOL (Secretaría de Desarrollo Social), 2014. Reglas de operación del programa para el desarrollo de zonas prioritarias (PDZP), para el ejercicio fiscal 2014. Publicado en el Diario Oficial de la Federación el 28/12/2013. Disponible en: http://www.microrregiones.gob.mx/documentos/2014/RO_PDZP2014_DOF.pdf [Consultado 28/10/2014].

Szöllösi, R., I. Szöllösi-Varga, 2002. Total antioxidant power in some species of labiatae (adaptation of FRAP method). Acta Biologica Szegediensis 46: 125-127.

Turner, N.J., 1973. Plant taxonomic systems and ethnobotany of three contemporary indian groups of the Pacific Northwest (Haida, Bella Coola, and Lillooet). Tesis de Doctorado, University of British Columbia, Vancouver. Doi: 10.14288/1.0107164

Wen-Hsiung, K., T. Yi-Jung, L. Mei-Ju, C. Lih-Ling, 2010. Activity and characterization of secondary metabolites produced by a new microorganism for control of plant diseases. New Biotechnology 27: 397-402. Doi: 10.1016/j.nbt.2010.05.014

Wu, X., L. Gu, J. Holden, D.B. Haytowitz, S.E. Gebhardt, G. Beecher, R.L. Prior, 2004. Development of a database for total antioxidant capacity in foods: a preliminary study. Journal of Food Composition and Analysis 17: 407-422. Doi: 10.1016/j.jfca.2004.03.001 